\title{
Klebsiella pneumoniae multi-resistente, factores predisponentes y mortalidad asociada en un hospital universitario en Colombia
}

\author{
Lina M. Echeverri-Toro, Zulma V. Rueda, Wilmar Maya, Yuli Agudelo, Sigifredo Ospina
}

\section{Multidrug-resistant Klebsiella pneumoniae, predisposing factors and associated mortality in a tertiary-care hospital in Colombia}

Introduction: Bacterial resistance to antibiotics is a serious public health problem that is increasing worldwide. Resistant (R) Klebsiella pneumoniae is one of the main pathogens isolated in nosocomial infections. The aim of this study was to explore risk factors associated with the acquisition of infection by R-K. pneumoniae and mortality. Methods: Prospective cohort study conducted in a hospital of high complexity of Medellin, October/2009April/2010. The exposed group was defined as patients infected with R-K. pneumoniae (producing b-lactamases or carbapenemases). In order to identify risk factors associated with infection by R-K. pneumoniae and 30 day mortality, logistic regression and Cox proportional hazards regression were used. Results: 243 patients were included in the study, 84 infected with R-K. pneumoniae and 159 infected with susceptible K. pneumoniae. Female sex (OR = 2.51 95\% 1.37 to 4.6), the co-existence of cardiovascular disease (OR = 2.13 95\% CI 1.14 to 3.99), previous use of ceftriaxone (OR $=9.52$ 95\% CI 2.63 to 34.46) and carbapenems (OR = 4.23 95\% CI 2.41 to 7.42 ) were risk factors associated to infection with R-K. pneumoniae. Some predictors of mortality were malignant neoplasia (HR = 4.43 95\% CI 2.13 to 9.22) and mechanical ventilation (HR = 3.81 95\% CI 1.99 to 7.28). There was no difference in 30-day mortality when comparing patients in both groups. Conclusions: Female gender, cardiovascular disease and previous use of antimicrobials were associated with infection by R-K. pneumoniae. Thirty-day mortality was similar in both groups of patients.

Key words: Klebsiella pneumoniae, antimicrobial resistance, ESBL, carbapenemases, risk factors, mortality.

Palabras clave: Klebsiella pneumoniae, resistencia a antimicrobianos, $\beta$-lactamasas de espectro extendido, carbapenemasas, factores de riesgo, mortalidad.

\section{Introducción}

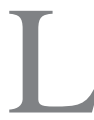
as enterobacterias comprenden universalmente el $50 \%$ de los aislados encontrados en infecciones adquiridas en los hospitales y 80\% de todos los aislados gramnegativos ${ }^{1}$. Dentro de esta familia, el segundo género en importancia es Klebsiella spp., siendo $K$. pneumoniae la especie más estudiada y de mayor relevancia clínica ${ }^{2,3}$. Datos del año 2009 recolectados en el Departamento de Antioquia, señalan a este microorganismo como el segundo agente encontrado en pacientes hospitalizados en unidades de cuidado intensivo (UCIs) cobrando gran importancia como causante de infecciones asociadas al cuidado de la salud ${ }^{4}$. Los datos publicados por el grupo GREBO de Bogotá, también para el 2009, reportan a K. pneumoniae como el segundo microorganismo aislado en pacientes hospitalizados ${ }^{2}$. Adicional al incremento en su prevalencia, se reporta un aumento de K. pneumoniae resistente a los antimicrobianos alrededor del mundo y en nuestro país ${ }^{2-4}$. Esta resistencia está dada principalmente por la producción de $\beta$-lactamasas de espectro extendido (BLEE) $)^{5,6}$ que inactivan a los antimicrobianos $\beta$-láctamicos, los más prescritos en todo el mundo ${ }^{7}$ y son el tratamiento de primera línea para las infecciones causadas por esta bacteria. Además, $K$. pneumoniae puede producir carbapenemasas que inactivan a los carbapenémicos, primera opción de tratamiento para las bacterias productoras de BLEE. Este tipo de resistencia se está convirtiendo en un problema de salud pública muy grave en todo el mundo ya que se agotan las opciones terapéuticas para los pacientes infectados con enterobacterias multi-resistentes ${ }^{8,9}$.

Los objetivos de este estudio fueron:

- Identificar los factores de riesgo asociados con la probabilidad de adquirir infección por K. pneumoniae resistente.

- Identificar los factores asociados con mortalidad en pacientes infectados con K. pneumoniae.
Universidad Pontificia Bolivariana, Medellín, Colombia (LME, ZVR) Hospital Universitario San Vicente de Paúl de Medellín, Colombia (WM, YA, SO)

Investigación realizada en el Hospital Universitario San Vicente de Paúl-Medellín.

Los autores manifiestan que no existe ningún conflicto de intereses. La investigación fue financiada por el Hospital Universitario San Vicente de Paúl de Medellín y la Universidad Pontificia Bolivariana.

Recibido: 20 de junio de 2011 Aceptado: 9 de enero de 2012

Correspondencia a: Lina María Echeverri Toro linamariae@hotmail.com 


\section{Materiales y Métodos}

\section{Población}

Estudio de cohorte fija que incluyó prospectivamente a todos los pacientes, de todas las edades, internados en el Hospital Universitario San Vicente de Paúl De Medellín entre el 10 de octubre de 2009 y 10 de abril de 2010, a quienes se les identificara $K$. pneumoniae como causante de infección.

Criterios de inclusión: aislamiento a partir de cualquier muestra biológica; primer aislamiento de K. pneumoniae identificado en cada paciente; infección adquirida en la comunidad o asociada al cuidado de la salud.

Criterios de exclusión: pacientes en quienes el aislamiento de $K$. pneumoniae fuera definido como colonizante y no tener acceso a la historia clínica.

Estudio aprobado por el Comité de Ética institucional.

\section{Procedimientos microbiológicos}

El médico tratante solicitaba los cultivos de las diferentes muestras biológicas (orina, sangre, líquidos estériles, hueso, etc.) ante la sospecha clínica de infección. Dichas muestras eran procesadas de acuerdo con los protocolos del laboratorio de la institución. En caso de detectar algún crecimiento bacteriano en los medios sólidos, agar sangre y/o agar MacConkey, o a partir de los hemocultivos, el aislado era llevado al equipo VITEK2® (bioMérieux, Inc. Hazelwood, MO, USA) para la identificación del microorganismo hasta especie y para la realización del antibiograma, determinando así los patrones de susceptibilidad a antimicrobianos de acuerdo con los puntos de corte establecidos por el Instituto de Estándares Clínicos y de Laboratorio de Estados Unidos de América (CLSI) ${ }^{10}$.

La producción de BLEE fue identificada por el sistema automatizado VITEK2 ${ }^{\circledR}$ (bioMérieux, Inc. Hazelwood, MO, USA), y la posible producción de carbapenemasas se sospechó por la presencia de resistencia a al menos uno de los siguientes carbapenémicos: imipenem, meropenem o ertapenem, y fue confirmada por el test modificado de Hodge, siguiendo las normas e indicaciones del CLSI $2009^{10}$.

\section{Variables recolectadas}

Los datos de interés para el estudio se tomaron de la historia clínica de cada paciente. Las variables evaluadas fueron co-morbilidades, tratamiento inmunosupresor, procedimiento quirúrgico durante la hospitalización, necesidad de ingreso a UCI, necesidad de ventilación mecánica, uso de catéter vesical, uso de catéter vascular central, uso previo de antimicrobianos en los últimos 30 días, muestra biológica donde se aisló el microorganismo, tiempo de estancia hospitalaria y mortalidad.

\section{Seguimiento y desenlace}

A todos los pacientes se les hizo seguimiento a los 7, 15 y 30 días a partir del aislado microbiológico. Las historias clínicas eran evaluadas en conjunto por un médico microbiólogo y un internista infectólogo, para determinar los aislados que no representaran infección. Si el paciente era dado de alta del hospital antes de completar el seguimiento, se le contactaba telefónicamente con el fin de evaluar el desenlace de interés, el cual fue mortalidad general a 30 días.

\section{Análisis estadístico}

El análisis se realizó usando el programa estadístico SPSS ${ }^{\circledR}$ versión 15.0 (SPSS Inc. Chicago, Il, USA). Se hizo distribución de frecuencias para describir las características generales de la población de estudio. Para determinar los factores asociados con la probabilidad de adquirir infección por K. pneumoniae resistente, se hizo un análisis de casos y controles, definiendo los casos como los pacientes con aislamiento de $K$. pneumoniae resistente, y los controles, los pacientes con aislamiento de K. pneumoniae sensible. Para esto se hizo una regresión logística múltiple. Los resultados son expresados como odds ratio (OR) con sus respectivos intervalos de confianza (IC) del 95\%. Para determinar los factores predictores de mortalidad a 30 días en los pacientes infectados por K. pneumoniae, se hicieron curvas de supervivencia de Kaplan-Meier y regresión de riesgos proporcionales de Cox. Para este análisis se tomaron dos grupos, uno de expuestos, definido como los pacientes con aislamiento de K. pneumoniae resistente, ya fuera por la producción de BLEE o de carbapenemasas, y otro grupo de no expuestos, definido como los pacientes con aislamiento de $\mathrm{K}$. pneumoniae sensible. Los resultados son expresados como hazard ratio (HR) con sus respectivos intervalos de confianza (IC) del 95\%. Para ambas regresiones, los criterios de entrada de las variables independientes fueron: que tuvieran un valor de $\mathrm{p}<0,25$ en el análisis bivariado o que en la literatura médica se hubieran descrito como factores de riesgo o protectores; las variables se ingresaron al modelo utilizando el método paso a paso. Por último se evaluó la prueba de bondad de ajuste de ambos modelos, y se consideró como significativo un valor $\mathrm{p}<0,05$.

\section{Resultados}

\section{Población de estudio}

En total se evaluaron 339 pacientes con aislamiento de K. pneumoniae, de los cuales ingresaron al estudio 243. La Figura 1 describe el flujograma de ingreso de pacientes. Del total de aislados, 159 fueron susceptibles y 84 resistentes. El fenotipo de resistencia más frecuente fue BLEE $(80 / 84=95 \%)$ y cuatro aislados fueron resistentes a carbapenémicos (5\%). De éstos, tres fueron 
aislados en sangre y uno en orina. La Tabla 1 muestra las características clínicas de la población de estudio.

Las principales infecciones causadas, tanto por el microorganismo sensible como resistente, fueron infección del tracto urinario y bacteriemias. El número total de infecciones urinarias en la cohorte fue 104, de los cuales 47 pacientes eran hombres y 57 mujeres.

En los pacientes con $K$. pneumoniae resistente se encontró mayor exposición a antimicrobianos (mínimo por 48 horas) en los últimos 30 días, al igual que estancias hospitalarias más prolongadas (Tabla 1 ).

Dos pacientes del total $(0,8 \%)$ tuvieron más de un aislamiento del microorganismo; sin embargo, se incluyeron en el estudio como episodios independientes debido a que ambos se captaron en hospitalizaciones diferentes y a que los fenotipos de susceptibilidad y el

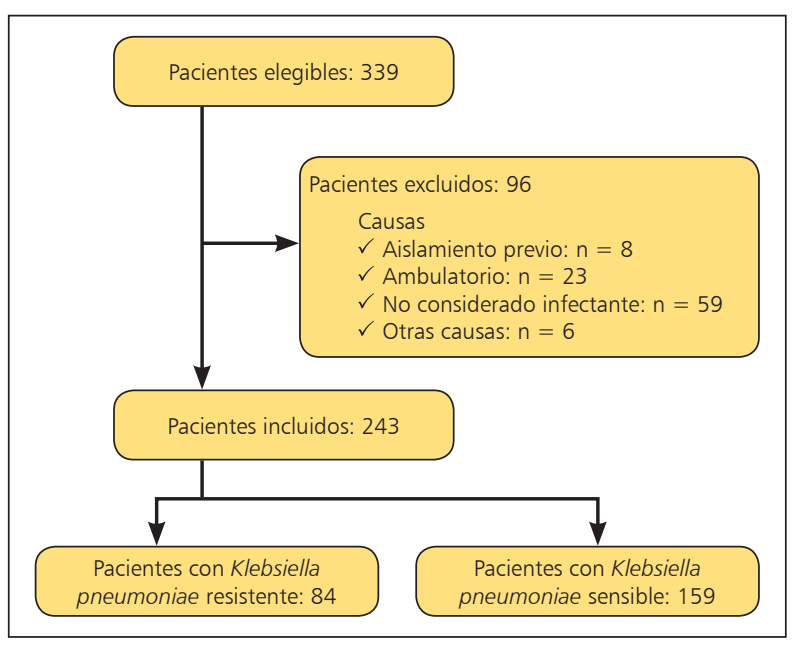

Figura 1. Flujograma de ingreso de pacientes al estudio.

Tabla 1. Características clínicas, microbiológicas y epidemiológicas de los pacientes infectados con K. pneumoniae sensible o resistente a antimicrobianos en un hospital universitario de Medellín 2009-2010

\section{Variables}

Edad en años, mediana (RIQ*)

Sexo, mujer (\%)

Co-morbilidades, (\%):

- Enfermedad pulmonar crónica

- Trasplante de órgano sólido

- Neoplasia maligna

- Diabetes mellitus

- Enfermedad renal crónica

- Enfermedad cardiovascular crónica

- Trauma penetrante de abdomen últimos 30 días

Procedimiento quirúrgico durante la hospitalización, (\%)

Tratamiento inmunosupresor durante la hospitalización, (\%)

Muestra biológica donde se aisló K. pneumoniae, (\%):

- Sangre

- Orina

- Muestras respiratorias

- Líquido peritoneal

- Tejido (hueso)

- Punta de catéter

- Secreciones y pus

Uso de catéter vesical, (\%)

Infección asociada al cuidado de la salud, (\%)

Uso de antimicrobianos durante la actual hospitalización en los 30 días previos (mayor a 48 horas) a la solicitud del cultivo, (\%)

Necesidad de $\mathrm{UCl}^{* *}$, (\%)

Necesidad de ventilación mecánica, (\%)

Estancia hospitalaria total en días, (\%)

Menor a 30 días

Mayor a 30 días

Mortalidad, (\%)
Pacientes con

K. pneumoniae sensible

$(n=159)$

$43(18-63)$

$59(37,1)$

$35(2,2)$

$4(2,5)$

$21(13,2)$

$22(13,8)$

$18(11,3)$

$43(27)$

$6(3,8)$

$84(52,8)$

$15(9,4)$

$33(20,8)$

$64(40,3)$

$25(15,7)$

$14(8,8)$

$9(5,7)$

$2(1,2)$

$14(8,8)$

$103(64,8)$

$68(42,8)$

$53(33,3)$

$75(47,2)$

$63(39,6)$

$100(62,9)$

$50(31,4)$

$30(18,9)$
Pacientes con

K. pneumoniae resistente $(n=84)$

$50(16,25-62,75)$

$48(57,14)$

$17(20,2)$

$3(3,6)$

$10(11,9)$

$17(20,2)$

$19(22,6)$

$35(41,7)$

0 (0)

$45(53,6)$

$15(17,9)$

21 (25)

$40(47,6)$

$8(9,5)$

$3(3,6)$

$10(11,9)$

$1(0,84)$

$1(0,84)$

$60(71,4)$

$44(52,4)$

$54(64,3)$

$31(36,9)$

26 (31)

$35(41,7)$

$43(51,2)$

$12(14,3)$

*RIQ: Rango intercuartílico ** UCl: Unidad de cuidados intensivos. 
tipo de infección que motivó cada hospitalización fueron distintos. Además, se consideró que la infección previa estaba resuelta, porque tenían los cultivos de control negativos y habían recibido el tiempo de antibioticoterapia indicado para cada caso. Por esta razón, el número total de muestras en el grupo de pacientes con K. pneumoniae sensible fue de 161 .

\section{Factores asociados con la probabilidad de adquirir infección por $K$. pneumoniae resistente a antimicrobianos}

El análisis bivariado muestra que los pacientes con diabetes mellitus (OR = 2,29 IC 95\% 1,12-4,65) y con enfermedad cardiovascular (OR = 1,92 IC 95\% 1,10-3,36) tuvieron un mayor riesgo de adquirir infección por $K$. pneumoniae resistente ( $\mathrm{p}<0,05)$. Así mismo, se encontró que el uso de ceftriaxona (OR = 9,52 IC 95\% 2,63-34,46) y carbapenémicos (OR = 4,23 IC95\% 2,41-7,42) mínimo por 48 horas, en los últimos 30 días previos al aislamiento del microorganismo resistente fueron factores de riesgo, pero no se encontró asociación con la exposición previa a ampicilina/sulbactam, aztreonam, aminoglucósidos, quinolonas ni piperacilina/tazobactam (Tabla 2).

El análisis multivariado mostró que las mujeres
(OR=2,51 IC 95\% 1,37-4,6), los pacientes con enfermedad cardiovascular de base (principalmente hipertensión arterial) (OR= 2,13 IC 95\% 1,14-3,99) y con exposición previa a ceftriaxona (OR 7,78 IC 95\% 2-30) y carbapenémicos (OR 4,19 IC 95\% 2,27-7,72), tienen mayor probabilidad de adquirir infección por $K$. pneumoniae resistente (Tabla 3). No se encontró asociación entre riesgo de adquirir infección por $K$. pneumoniae resistente y necesidad de ventilación mecánica, ingreso a UCI, uso de catéter vesical, tratamiento inmunosupresor ni neoplasia maligna previo al aislamiento.

\section{Supervivencia a 30 días en pacientes infectados con $K$. pneumoniae y factores predictores de mortalidad}

La Figura 2 muestra las curvas de supervivencia entre los pacientes infectados con $K$. pneumoniae sensible y resistente, en la cual se observa que no hubo diferencias en la mortalidad a 30 días entre ambos grupos (valor p de Log Rank = 0,22). Se encontró una mayor mortalidad en los pacientes que tenían enfermedad cardiovascular, enfermedad pulmonar crónica, neoplasia maligna, bacteriemia, aquellos que ingresaban a la UCI o que requerían ventilación mecánica durante la hospitalización,

\section{Tabla 2. Análisis bivariado. Factores asociados con el riesgo de adquirir infección por Klebsiella pneumoniae resistente a antimicrobianos}

\begin{tabular}{|c|c|c|c|c|c|}
\hline Variables & $\begin{array}{c}\text { Pacientes con } \\
\text { K. pneumoniae sensible } \\
(\mathrm{n}=159)\end{array}$ & $\begin{array}{c}\text { Pacientes con } \\
\text { K. pneumoniae resistente } \\
(\mathrm{n}=84)\end{array}$ & OR & IC $95 \%$ & Valor de $p$ \\
\hline Sexo, mujer & 59 & 48 & 2,26 & $1,31-3,87$ & $<0,001$ \\
\hline $\begin{array}{l}\text { Co-morbilidades: } \\
\text { - Trasplante de órgano sólido } \\
\text { - Diabetes mellitus } \\
\text { - Enfermedad cardiovascular } \\
\text { - Enfermedad pulmonar crónica } \\
\text { - Neoplasia maligna } \\
\text { - Tratamiento inmunosupresor } \\
\text { - Enfermedad renal crónica }\end{array}$ & $\begin{array}{r}4 \\
18 \\
43 \\
35 \\
21 \\
15 \\
22\end{array}$ & $\begin{array}{r}3 \\
19 \\
35 \\
17 \\
10 \\
15 \\
17\end{array}$ & $\begin{array}{l}1,43 \\
2,29 \\
1,92 \\
0,89 \\
0,88 \\
0,47 \\
1,58\end{array}$ & $\begin{array}{l}0,31-6,56 \\
1,12-4,65 \\
1,10-3,36 \\
0,46-1,72 \\
0,39-1,98 \\
0,22-1,03 \\
0,78-3,17\end{array}$ & $\begin{array}{l}0,69 \\
0,02 \\
0,02 \\
0,74 \\
0,77 \\
0,05 \\
0,19\end{array}$ \\
\hline $\begin{array}{l}\text { Uso de antimicrobianos por más de } 48 \text { horas en los } 30 \text { días } \\
\text { previos a la toma del cultivo } \\
\text { - ceftriaxona } \\
\text { - aminoglucósido } \\
\text { - quinolonas } \\
\text { - } \text { ampicilina/sulbactam o aztreonam } \\
\text { - piperacilina/tazobactam } \\
\text { - carbapenémicos (meropenem, imipenem y/o ertapenem) }\end{array}$ & $\begin{array}{r}3 \\
21 \\
31 \\
57 \\
53 \\
41\end{array}$ & $\begin{array}{l}13 \\
14 \\
18 \\
25 \\
32 \\
50\end{array}$ & $\begin{array}{l}9,52 \\
1,31 \\
1,12 \\
0,75 \\
1,23 \\
4,23\end{array}$ & $\begin{array}{l}2,63-34,46 \\
0,63-2,74 \\
0,58-2,16 \\
0,42-1,34 \\
0,71-2,13 \\
2,41-7,42\end{array}$ & $\begin{array}{c}<0,001 \\
0,46 \\
0,72 \\
0,34 \\
0,45 \\
<0,001\end{array}$ \\
\hline $\begin{array}{l}\text { Ingreso a UCI** durante la hospitalización previo a la solicitud } \\
\text { del cultivo donde se aisló } K \text {. pneumoniae }\end{array}$ & $67 / 75^{*}$ & $28 / 31 *$ & 1,11 & $0,27-4,51$ & 1,00 \\
\hline $\begin{array}{l}\text { Ventilación mecánica previo a la solicitud del cultivo donde se } \\
\text { aisló } K \text {. pneumoniae }\end{array}$ & $18 / 63^{*}$ & $6 / 26^{*}$ & 0,75 & $0,25-2,17$ & 0,59 \\
\hline $\begin{array}{l}\text { Uso de catéter vesical previo a la solicitud del cultivo donde se } \\
\text { aisló } K \text {. pneumoniae }\end{array}$ & $31 / 103^{*}$ & $11 / 60^{*}$ & 0,52 & $0,24-1,13$ & 0,09 \\
\hline Infección asociada al cuidado de la salud & 68 & 44 & 1,51 & $0,88-2,57$ & 0,12 \\
\hline
\end{tabular}


Tabla 3. Análisis multivariado. Factores asociados con la probabilidad de adquirir infección por Klebsiella pneumoniae resistente a antimicrobianos

\begin{tabular}{|c|c|c|c|c|c|}
\hline Variable & Coeficiente & Error estándar & Test de Wald & OR & IC $95 \%$ del OR \\
\hline Sexo femenino & 0,92 & 0,31 & 8,81 & 2,51 & $1,37-4,6$ \\
\hline Enfermedad cardiovascular & 0,76 & 0,32 & 5,59 & 2,13 & $1,14-3,99$ \\
\hline Uso de ceftriaxona por más de 48 horas previas a la toma del cultivo & 2,05 & 6,93 & 8,77 & 7,78 & $2,00-30,25$ \\
\hline $\begin{array}{l}\text { Uso de carbapenémicos (imipenem, meropenem o ertapenem) por más de } 48 \text { horas } \\
\text { previas a la toma del cultivo }\end{array}$ & 1,43 & 0,31 & 21,08 & 4,19 & $2,27-7,72$ \\
\hline
\end{tabular}

comparado con los que no presentaban estas condiciones (Tabla 4). No se encontró diferencia en la mortalidad por género $(\mathrm{p}=0,12)$.

En el análisis multivariado se encontró que los factores asociados con un mayor riesgo de muerte fueron enfermedad cardiovascular, enfermedad pulmonar crónica, neoplasias malignas, bacteriemia y la necesidad de ventilación mecánica durante la hospitalización (Tabla 5).

\section{Discusión}

Los bacilos gramnegativos entéricos resistentes a antimicrobianos son un problema frecuente en todo el mundo y han cobrado gran importancia como causantes de

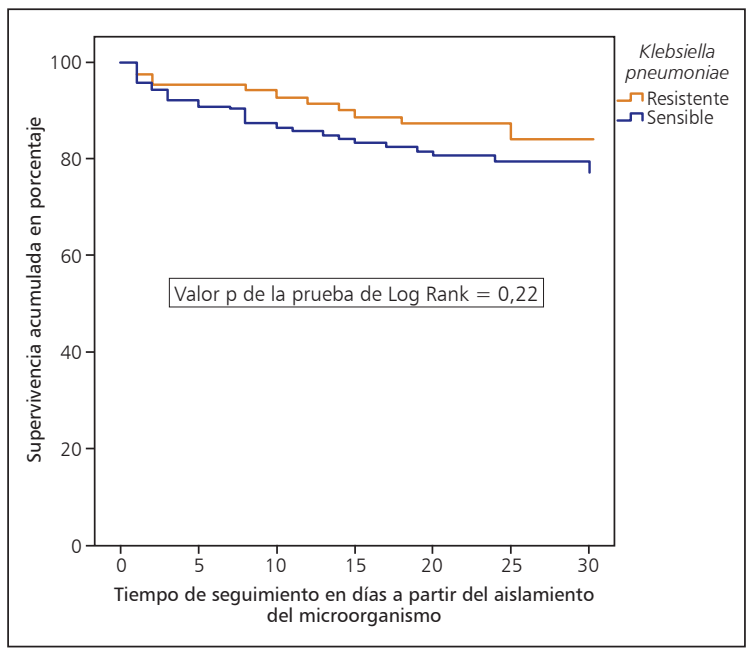

Figura 2. Curvas de supervivencia de los pacientes infectados con K. pneumoniae sensible y resistente, hospitalizados en una institución de tercer nivel de Medellín, 2009-2010.

\begin{tabular}{|c|c|c|c|}
\hline Variables (condición) & $\begin{array}{l}\text { Mortalidad en quienes presen- } \\
\text { tan la condición }\end{array}$ & $\begin{array}{l}\text { Mortalidad en quienes no pre- } \\
\text { sentan la condición }\end{array}$ & $\begin{array}{l}\text { Valor } p \\
\text { Log rank }\end{array}$ \\
\hline \multicolumn{4}{|l|}{ Co-morbilidades: } \\
\hline Enfermedad pulmonar crónica & $17 / 52$ & $25 / 189$ & $<0,001$ \\
\hline Neoplasia maligna & $12 / 30$ & $30 / 211$ & $<0,001$ \\
\hline Diabetes mellitus & $7 / 37$ & $35 / 204$ & 0,86 \\
\hline Enfermedad renal crónica & $7 / 39$ & $35 / 202$ & 0,97 \\
\hline Enfermedad cardiovascular crónica & $22 / 78$ & $20 / 163$ & $<0,001$ \\
\hline Bacteriemia & $19 / 53$ & $23 / 188$ & $<0,001$ \\
\hline Infección del tracto urinario & $9 / 104$ & $33 / 137$ & 0,003 \\
\hline Infección del tracto respiratorio inferior & 9/ 32 & $33 / 209$ & 0,052 \\
\hline Peritonitis & 4/ 17 & $38 / 224$ & 0,499 \\
\hline Infección en otros líquidos estériles & $3 / 4$ & $39 / 237$ & $<0,001$ \\
\hline Necesidad de ingreso a UCl** & 29/105 & $13 / 136$ & $<0,001$ \\
\hline Necesidad de ventilación mecánica & $26 / 88$ & $16 / 153$ & $<0,001$ \\
\hline Estancia hospitalaria > 30 días a partir del diagnóstico de $K$. pneumoniae & $12 / 73$ & $20 / 48$ & $<0,001$ \\
\hline
\end{tabular}


Tabla 5. Análisis multivariado. Factores predictores asociados con la mortalidad a 30 días en pacientes hospitalizados e infectados con Klebsiella pneumoniae

\begin{tabular}{|c|c|c|c|c|c|}
\hline Variable & Coeficiente beta & Error estándar del coeficiente & Test de Wald & HR & IC $95 \%$ del HR \\
\hline Enfermedad pulmonar crónica & 1,013 & 0,35 & 8,31 & 2,76 & $1,38-5,48$ \\
\hline Neoplasia maligna & 1,489 & 0,37 & 15,85 & 4,43 & $2,13-9,22$ \\
\hline Enfermedad cardiovascular & 0,806 & 0,34 & 5,56 & 2,24 & $1,16-4,37$ \\
\hline Bacteriemia por K. pneumoniae & 1,118 & 0,31 & 12,35 & 3,06 & $1,64-5,71$ \\
\hline Necesidad de ventilación mecánica & 1,338 & 0,33 & 16,34 & 3,81 & $1,99-7,28$ \\
\hline
\end{tabular}

infecciones asociadas al cuidado de la salud ${ }^{2,3}$. Latinoamérica no se escapa a esta realidad; en diferentes ciudades de Colombia y en nuestra institución se ha documentado en los últimos años un aumento en el aislamiento de estos microorganismos, especialmente aquellos productores de BLEE como K. pneumoniae. Lo anterior motivó la realización del presente trabajo de investigación, siendo el hallazgo más relevante que el uso de ceftriaxona y carbapenémicos en los 30 días previos al aislamiento de $K$. pneumoniae resistente, es un factor de riesgo para adquirir infección por esta bacteria. No hubo diferencias en la mortalidad entre los pacientes infectados por $K$. pneumoniae sensible y resistente.

Respecto al primer hallazgo, una serie de investigaciones han demostrado fuerte asociación entre el uso previo de antimicrobianos y la infección por microorganismos resistentes productores de BLEE, probablemente debido a que éstos ejercen presión selectiva eliminando las cepas sensibles a antimicrobianos en el individuo expuesto ${ }^{11-13}$. Después de la aparición de los primeros microorganismos productores de esas enzimas en 1983, Meyer y cols., describieron que el uso previo de ceftazidima se asoció con la posibilidad de aislar microorganismos resistentes ${ }^{14}$. Posteriormente, Quale y cols. ${ }^{15}$, en un estudio realizado en 2002 en 15 hospitales en la ciudad de Brooklyn (Nueva York), concluyeron que el uso de cefalosporinas asociadas a aztreonam se correlacionó directamente con la presencia de BLEE en los microorganismos estudiados $(p=0,014)$. Peña y cols. ${ }^{16}$, reportaron resultados similares, en un estudio realizado en España donde la relación más frecuente con la presencia de microorganismos productores de BLEE fue el uso de cefalosporinas de tercera generación y aztreonam, al igual que lo reportado por Paterson en $2005^{17}$.

En el presente estudio se evaluaron varios grupos de antimicrobianos (quinolonas, aminoglucósidos, $\beta$ lactámicos) de manera prospectiva y sólo encontramos asociación significativa con el uso previo de ceftriaxona y de carbapenémicos, con los cuales se encontró un aumento de siete y cuatro veces, respectivamente, el riesgo de infección por $K$. pneumoniae productora de
BLEE, comparado con aquellos que no habían estado expuestos a estos medicamentos. Esto contrasta con otros estudios, retrospectivos, en los cuales los antimicrobianos asociados al riesgo de adquirir microorganismos productores de BLEE han sido quinolonas, monobactámicos, aminoglucósidos y más recientemente, piperacilina/ tazobactam, hallazgo reportado por Vargas y cols., en un estudio realizado en Brasil ${ }^{18}$. Cabe anotar que en el caso de aztreonam, posiblemente no encontramos diferencias debido a su bajo uso en nuestra institución.

En este estudio encontramos asociación entre la infección por $K$. pneumoniae resistente y el uso de carbapenémicos, lo cual puede explicarse porque el uso de estos antimicrobianos produce un cambio en la microbiota gastrointestinal que favorece la presencia de microorganismos productores de BLEE, como está descrito por algunos autores. La asociación entre la presencia de BLEE y el uso de algunos antimicrobianos demuestra la importancia de implementar intervenciones dirigidas a restringir su uso. Por ejemplo, Peña ${ }^{16}$, en un estudio publicado en 2001, demostró que la restricción del uso de cefalosporinas de espectro extendido en las UCIs de un hospital de Barcelona, permitió controlar un brote de K. pneumoniae productora de BLEE, lo cual está a favor de implementar políticas de restricción del uso de antimicrobianos en las instituciones de salud.

Otro hallazgo relevante de este estudio es que la mortalidad general en ambos grupos de pacientes fue similar. Esto es contrario a lo descrito por un sinnúmero de artículos que abordan este tema, los cuales en su mayoría fueron realizados antes del año 2000, e indican que estar infectado con microorganismos multi-resistentes conlleva una mayor mortalidad ${ }^{19}$. Este hallazgo podría ser explicado porque las condiciones de nuestra institución permiten hacer un diagnóstico rápido, asociado a un inicio temprano de antimicrobianos que son dirigidos específicamente contra el microorganismo aislado como causante de infección, sumado al adecuado manejo de las co-morbilidades. Algunos autores han descrito que estas variables se asocian con un mejor desenlace en este tipo de pacientes $^{5,20,21}$. Otra posible explicación para este hallazgo 
es que los microorganismos productores de BLEE tengan menor virulencia.

$\mathrm{Al}$ evaluar las características clínicas que se asociaron con un mayor riesgo de tener infección por K. pneumoniae resistente se encontraron la enfermedad cardiovascular y la diabetes mellitus (DM). Esta última asociación ha sido reportada en la literatura científica mundial y sugiere que la presencia de esta infección no sólo se relaciona con los factores biológicos y de virulencia inherentes y adquiridos por este microorganismo, sino que se relaciona también con los factores biológicos e inmunes propios del hospedero ${ }^{1,11,20-23}$. Sin embargo, en el análisis multivariado solo se encontró asociación con enfermedad cardiovascular.

Respecto a este resultado, consideramos que es un hallazgo incidental de nuestro estudio, pues no encontramos una explicación biológica plausible y tampoco encontramos reportes de esta asociación en la literatura; por lo tanto, proponemos que este hallazgo sea corroborado con estudios posteriores.

El análisis multivariado también mostró que el sexo femenino está asociado con la probabilidad de adquirir infección por K. pneumoniae resistente, dato no reportado previamente en la literatura médica revisada y que podría ser explicado por la mayor prevalencia de las infecciones urinarias en este género y el subsecuente uso de antimicrobianos empíricos para su tratamiento.

Para otras variables evaluadas, como uso de catéter intravascular, uso de catéter vesical, necesidad de ventilación mecánica, entre otras, no se encontró asociación con la infección por microorganismos productores de BLEE, quizá por las diferentes medidas estandarizadas y de control que se toman en todo el mundo con estos dispositivos desde hace unos años, lo cual está a favor de que se presente un menor número de infecciones asociadas a estas condiciones. Cabe anotar que los hallazgos anteriores van en contra de otros estudios publicados, los cuales señalan a estas variables como factores de riesgo para adquirir esta infección.

En nuestro estudio tampoco se encontró asociación entre infección por K. pneumoniae resistente con el trasplante de órgano sólido, ni con la exposición a tratamiento inmunosupresor, posiblemente debido al pequeño número de pacientes ingresados que tenían alguna de estas dos condiciones de base.

El análisis de los resultados permite concluir que no existe diferencia en la mortalidad a 30 días entre los pacientes infectados con el microorganismo resistente, al compararlos con los infectados con el microorganismo sensible. Autores como Tumbarello y cols., reportaron en el año 2006, una mayor mortalidad en los pacientes infectados por microorganismos productores de BLEE, asociada con el inadecuado uso de terapia antimicrobiana ${ }^{24}$. Se sugiere un estudio posterior que evalúe la mortalidad atribuible a la infección por Klebsiella multi-resistente.
El presente estudio da pie para proponer futuras investigaciones de tipo molecular, con el fin de hacer genotipificación de las cepas resistentes a antimicrobianos y determinar si existe evidencia de relación clonal entre cepas, para confirmar la presencia o no de transmisión cruzada. También da pie para realizar estudios que evalúen el impacto en los costos que tienen las infecciones por microorganismos resistentes. Algunas limitaciones de este estudio fueron: no se evaluó la historia de infecciones previas y recientes que hubieran requerido tratamiento antimicrobiano; no se evaluaron los microorganismos resistentes productores de $\beta$-lactamasas tipo AmpC, debido a la dificultad para su confirmación con las técnicas convencionales del laboratorio. Por lo tanto, podrían existir dentro del estudio aislados catalogados como sensibles aunque pudieran tener este tipo de resistencia; y el seguimiento de este estudio se hizo a 30 días; no obstante, algunos estudios sugieren que debe ser más prolongado con el fin de evaluar si la infección por microorganismos resistentes tiene influencia en la prolongación de la estancia hospitalaria.

\section{Conclusión}

El sexo femenino, la enfermedad cardiovascular y el uso previo de antimicrobianos como ceftriaxona y carbapenémicos están asociados con infección por el microorganismo resistente, por lo tanto, la restricción del uso de dichos antimicrobianos podría contribuir a evitar la aparición de microorganismos productores de BLEE.

El presente estudio demuestra que la mortalidad general en ambos grupos de pacientes fue similar.

Agradecimientos. Al equipo de bacteriólogas (os) de la sección de Microbiología del Laboratorio del Hospital, a Jaime Robledo y a Jaime López por su asesoría inicial en este trabajo y al Hospital Universitario San Vicente de Paúl Medellín.

\section{Resumen}

Introducción: La resistencia bacteriana a antimicrobianos es un grave problema de salud pública que va aumentando en el mundo. Klebsiella pneumoniae resistente $(\mathrm{R})$ es uno de los principales patógenos aislado en infecciones hospitalarias. El objetivo de este estudio fue explorar factores de riesgo asociados con la adquisición de infección por K. pneumoniae $\mathrm{R}$ y con mortalidad. Metodología: Estudio de cohorte prospectivo realizado en un hospital de alta complejidad de Medellín, octubre/2009-abril/2010. El grupo expuesto se definió como pacientes infectados por K. pneumoniae $\mathrm{R}$ (productora de $\beta$-lactamasas de espectro extendido o carbapenemasas). Se hicieron regresión logística para identificar los factores 
de riesgo asociados con infección por $K$. pneumoniae $\mathrm{R}$, y regresión de riesgos proporcionales de Cox para identificar los factores asociados con mortalidad a 30 días. Resultados: Se incluyeron 243 pacientes al estudio, 84 infectados con K. pneumoniae R y 159 con K. pneumoniae sensible.El sexo femenino (OR = 2,51 IC95\% 1,37-4,6), la co-existencia de enfermedad cardiovascular (OR = 2,13 IC 95\% 1,14-3,99), uso previo de ceftriaxona (OR = 9,52 IC95\% 2,63-34,46) y carbapenémicos (OR = 4,23 IC95\% 2,41-7,42) fueron factores de riesgo asociados con la probabilidad de infectarse por K. pneumoniae R. Algunos factores predictores de mortalidad fueron las neoplasias malignas (HR $=4,43$ IC95\% 2,13-9,22) y la ventilación mecánica (HR = 3,81 IC95\% 1,99-7,28). No hubo diferencia en la mortalidad a 30 días al comparar los pacientes de ambos grupos. Conclusiones: El sexo femenino, la enfermedad cardiovascular y el uso previo de antimicrobianos se vieron asociados con infección por K. pneumoniae R. La mortalidad a 30 días fue similar en ambos grupos de pacientes.

\section{Referencias}

1.- Abbot S L. Klebsiella, Enterobacter, Citrobacter, Serratia, Plesiomonas, and other Enterobacteriaceae. En: Murray PR, editor. Manual of Clinical Microbiology. 9 ed. Washington: ASM Press; 2007. p. 698-711.

2.- Leal A, Schmalbach J, Álvarez C, Buitrago G, Méndez M. Canales endémicos y marcadores de resistencia bacteriana en instituciones de tercer nivel de Bogotá, Colombia. Rev Salud Pública. 2006; 8 (1): 59-70.

3.- Hoyos-Orrego A, Rivera-Rivera O, HoyosPosada C, Mesa-Restrepo C, Alfaro-Velásquez J. Características clínicas, epidemiológicas y de susceptibilidad a los antibióticos en casos de bacteriemia por Klebsiella pneumoniae en neonatos. Rev CES Med 2007; 21 (2): 31-9.

4.- Grupo para el Estudio de la Resistencia a Antibióticos de Medellín. Perfiles de sensibilidad de Klebsiella pneumoniae [sede web]. Medellín: Germen. 2009 [consultado 1 de abril de 2010]. Disponible en: http://www. grupogermen.org

5.- Peña C, Pujol M, Ardanuy C, Ricart A, Pallares J, Linares J, et al. An outbreak of hospitalacquired Klebsiella pneumoniae bacteraemia, including strains producin extended-spectrum beta-lactamases. J Hosp Infec 2001; 47: 53-9.

6.- Navarro M, Moreno B, López B, Fragoso M. Detección de cepas de Escherichia coli y Klebsiella pneumoniae productoras de betalactamasas de espectro extendido (BLEE) en el Hospital Infantil del Estado de Sonora. Bol Clin Hosp Infant Edo Son 2005; 22 (2): 64-70.

7.- Rahal J J, Urban C, Horn D, Freeman K, Segal-Maurer S, Maurer J, et al. Class restriction of cephalosporin use to control total cephalosporin resistance in nosocomial Klebsiella. JAMA 1998; 280 (14): 1233-7.

8.- Karthikeyan K, Toleman M, Walsh T, Bagaria J, Butt F, Balakrishnan R, et al. Emergence of a new antibiotic resistance mechanism in India, Pakistan, and the UK: a molecular, biological, and epidemiological study Lancet 2010. Publicado online agosto 11, 2010 DOI: 10.1016/
S1473-3099(10)70143-2).

9.- Deshpande P, Rodrigues C, Shetty A, Kapadia F, Hedge A, Soman R. New Delhi metallo- $\beta$ lactamase (NDM-1) in Enterobacteriaceae: Treatment options with carbapenems compromised. J Assoc Physicians India. 2010; 58: $147-9$

10.- CLSI. Performance Standards for Antimicrobial Susceptibility Testing; nineteenth informational supplement, CLSI document M100-S19. Wayne, PA: Clinical Laboratory Standards Institute; 2009. p. 1-158.

11.- Lautenbach E, Patel J B, Bilker W B, Edelstein P H, Fishman N O. Extended spectrum betalactamase producing Escherichia coli and Klebsiella pneumoniae: risk factors for infection and impact of resistance on outcomes. Clin Infect Dis 2001; 32 (8): 1162-71.

12.- Du B, Long Y, Liu H, Chen D, Liu D, Xu Y, et al. Extended-spectrum beta-lactamase producing Escherichia coli and Klebsiella pneumoniae bloodstream infection: risk factors and clinical outcome. Intensive Care Med 2002; 28 (12): 1718-23.

13.- Paterson D, Ko W, Gottberg A, Mohapatra S, Casellas J, Goossens H, et al. International prospective study of Klebsiella pneumoniae bacteremia: implications of extended-spectrum beta-lactamase production in nosocomial infections. Ann Intern Med 2004; 140: 26-32.

14.- Meyer K, Urban C, Eagan J, Berger B, Rahal J. Nosocomial outbreak of Klebsiella infection resistant to late-generation cephalosporins. Ann Intern Med 1993; 119 (5): 353-8.

15.- Quale J, Bradford P, Visalli M, Ravishankar J, Flores C, Mayorga D, et al. Molecular epidemiology of a citywide outbreak of extended-spectrum beta-lactamase-producing Klebsiella pneumoniae infection. Clin Infect Dis 2002; 35 (7): 834-41.

16.- Peña C, Pujol M, Ardanuy C, Ricart A, Pallares J, Linares J, et al. An outbreak of hospitalacquired Klebsiella pneumoniae bacteraemia, including strains producin extended-spectrum beta-lactamase. J Hosp Infec 2001; 47: 53-9.

17.- Paterson D y Bonomo R. Extended-spectrum beta-lactamases: a clinical update. Clin Microb Rev 2005; 18 (4): 657-86.

18.- Vargas S, Augusti G y Zavascki A. Risk factors for and mortality of extended spectrum $\beta$-lactamase-producing Klebsiella pneumoniae and Escherichia coli nosocomial bloodstream infections. Rev Inst Med Trop. S. Paulo 2009; 51 (4): 211-6.

19.- Chow J W, Fine M J, Shlaes D M, Quinn J P, Hooper D C, Johnson M P, et al. Enterobacter bacteremia: clinical features and emergence of antibiotic resistance during therapy. Ann Intern Med 1991; 115 (8): 585-90.

20.- Marra A, Wey S B, Castelo A, Gales A C, Cal R G, Filho J R, et al. Nosocomial bloodstream infections caused by Klebsiella pneumoniae: impact of extended-spectrum beta-lactamase (ESBL) production on clinical outcome in a hospital with high ESBL prevalence. BMC Infect Dis 2006; 14: 6-24.

21.- Kang C I, Kim S H, Park W B, Lee K D, Kim H B, Kim E C, et al. Bloodstream infections due to extended-spectrum betalactamase producing Escherichia coli and Klebsiella pneumoniae: risk factors for mortality and treatment outcome, with special emphasis on antimicrobial therapy. Antimicrob Agents Chemother 2004; 48 (12): 4574-81.

22.- Gupta A, Ampofo K, Rubenstein D, Saiman L. Extended spectrum beta lactamase producing Klebsiella pneumoniae infections: a review of the literature. J Perinatol 2003; 23: 439-43.

23.- Sahly H, Navon-Venezia S, Roesler L, Hay A, Carmeli Y, Podschun R, et al. Extendedspectrum beta-lactamase production is associated with an increase in cell invasion and expression of fimbrial adhesins in Klebsiella pneumoniae. Antimicrob Agents Chemother 2008; 52 (9): 3029-34.

24.- Tumbarello M, Spanu T, Sanguinetti M, Citton $\mathrm{R}$, Montuori E, Leone F, et al. Bloodstream infections caused by extended spectrum ß-lactamase-producing Klebsiella pneumoniae: Risk factors, molecular epidemiology, and clinical outcome. Antimicrob Agents Chemother 2006; 50 (2): 498-504. 